\title{
ON WELL-POSEDNESS, REGULARITY AND ILL-POSEDNESS FOR THE NONLINEAR FOURTH-ORDER SCHRÖDINGER EQUATION
}

\author{
VAN DuOng Dinh
}

\begin{abstract}
We prove the local well-posedness for the nonlinear fourth-order Schrödinger equation (NL4S) in Sobolev spaces. We also studied the regularity of solutions in the sub-critical case. A direct consequence of this regularity is the global well-posedness above mass and energy spaces under some assumptions. Finally, we show the ill-posedness for (NL4S) in some cases of the super-critical range.
\end{abstract}

\section{Introduction and main results}

We consider the Cauchy fourth-order Schrödinger equation posed on $\mathbb{R}^{d}, d \geq 1$, namely

$$
\left\{\begin{aligned}
i \partial_{t} u(t, x)+\Delta^{2} u(t, x) & =-\mu|u|^{\nu-1} u(t, x), \quad(t, x) \in \mathbb{R} \times \mathbb{R}^{d}, \\
u(0, x) & =u_{0}(x), \quad x \in \mathbb{R}^{d} .
\end{aligned}\right.
$$

where $\nu>1$ and $\mu \in\{ \pm 1\}$. The number $\mu=1$ (resp. $\mu=-1$ ) corresponds to the defocusing case (resp. focusing case).

The fourth-order Schrödinger equation was introduced by Karpman 20] and Karpman and Shagalov [21] concerning the role of small fourth-order dispersion terms in the propagation of intense laser beams in a bulk medium with Kerr nonlinearity. The study of nonlinear fourthorder Schrödinger equation has been attracted a lot of interest in a past decay (see [26], 27], [16], 17], 19] and references cited therein).

It is worth noticing that if we set for $\lambda>0$,

$$
u_{\lambda}(t, x)=\lambda^{-\frac{4}{\nu-1}} u\left(\lambda^{-4} t, \lambda^{-1} x\right)
$$

then the (NL4S) is invariant under this scaling. An easy computation shows

$$
\left\|u_{\lambda}(0)\right\|_{\dot{H}^{\gamma}}=\lambda^{\frac{d}{2}-\frac{4}{\nu-1}-\gamma}\|\varphi\|_{\dot{H}^{\gamma}}
$$

From this, we define the critical regularity exponent for (NL4S) by

$$
\gamma_{c}=\frac{d}{2}-\frac{4}{\nu-1}
$$

One said that $H^{\gamma}$ is sub-critical (critical, super-critical) if $\gamma>\gamma_{c}\left(\gamma=\gamma_{c}, \gamma<\gamma_{c}\right)$ respectively. Another important property of (NL4S) is that the following mass and energy are formally conserved under the flow of the equation,

$$
M(u(t))=\int|u(t, x)|^{2} d x, \quad E(u(t))=\int \frac{1}{2}|\Delta u(t, x)|^{2}+\frac{\mu}{\nu+1}|u(t, x)|^{\nu+1} d x .
$$

The main purpose of this note is to give the well-posedness and ill-posedness results for (NL4S) in Sobolev spaces. In 9, the local well-posedness for the nonlinear fractional Schrödinger equation including the fourth-order Schrödinger equation in both sub-critical and critical cases are showed. We shall review the local well-posedness for the nonlinear fourth-order Schrödinger 
equation below. These results are very similar to the nonlinear Schrödinger equation given in [4]. We also give the local well-posedness in the critical Sobolev space $H^{d / 2}$. The global wellposedness in $L^{2}$ is then a direct consequence of the local existence and the conservation of mass. We also recall (see e.g. 26 or 9]) the global well-posedness in the energy space $H^{2}$ under some assumptions. We next show the regularity of solutions in the sub-critical case. As a consequence of this regularity, we obtain the global well-posedness above the mass and energy spaces for (NL4S) under some assumptions. The second part of this note is devoted to the ill-posedness of (NL4S). It is easy to see (e.g [24]) that the (NL4S) is ill-posed in $\dot{H}^{\gamma}$ for $\gamma<\gamma_{\mathrm{c}}$. Indeed if $u$ solves the (NL4S) with initial data $\varphi \in \dot{H}^{\gamma}$ with the lifespan $T$, then the norm $\left\|u_{\lambda}(0)\right\|_{\dot{H}^{\gamma}}$ and the lifespan of $u_{\lambda}$ go to zero as $\lambda \rightarrow 0$. Using the technique of Christ-Colliander-Tao given in [7, we are able to prove the ill-posedness for (NL4S) in some cases of the super-critical range, precisely in $H^{\gamma}$ with $\gamma \in\left((-\infty,-d / 2] \cap\left(-\infty, \gamma_{\mathrm{c}}\right)\right) \cup\left[0, \gamma_{\mathrm{c}}\right)$. This ill-posed result is similar to the nonlinear semi-relativistic equation given in [11. Note that for the nonlinear Schrödinger equation, the ill-posedness holds in $H^{\gamma}$ for $\gamma<\max \left\{0, \gamma_{c}\right\}$ (see [7]). The main difference is that the nonlinear Schrödinger equation has the Galilean invariance while (NL4S) does not share this property. The Galilean invariance plays a significant role in the proof of the ill-posedness in the range $\gamma \in(-d / 2,0)$. Recently, Hong and Sire in [18 used the pseudo-Galilean transformation to get the ill-posedness for the nonlinear fractional Schrödinger equation in Sobolev spaces of negative exponent. Unfortunately, it seems to be difficult to control the error of the pseudoGalilean transformation in high Sobolev norms and so far restricted in one dimension. We finally note that the well-posedness, regularity for the (NL4S) given in this note can be applied for the nonlinear fractional Schrödinger equation of order greater than or equal to 2 without any difficulty. Moreover, the ill-posedness argument can be adapted for the nonlinear fractional Schrödinger equation of any order.

Before stating our results, let us introduce some notations (see the appendix of [12, Chapter 5 of 31 or Chapter 6 of 3 ). Given $\gamma \in \mathbb{R}$ and $1 \leq q \leq \infty$, the generalized Sobolev space is defined by

$$
H_{q}^{\gamma}:=\left\{u \in \mathscr{S}^{\prime} \mid\|u\|_{H_{q}^{\gamma}}:=\left\|\langle\Lambda\rangle^{\gamma} u\right\|_{L^{q}}<\infty\right\}, \quad \Lambda=\sqrt{-\Delta},
$$

where $\langle\cdot\rangle$ is the Japanese bracket and $\mathscr{S}^{\prime}$ the space of tempered distributions. The generalized homogeneous Sobolev space is defined by

$$
\dot{H}_{q}^{\gamma}:=\left\{u \in \mathscr{S}_{0}^{\prime} \mid\|u\|_{\dot{H}_{q}^{\gamma}}:=\left\|\Lambda^{\gamma} u\right\|_{L^{q}}<\infty\right\}
$$

where $\mathscr{S}_{0}$ is a subspace of the Schwartz space $\mathscr{S}$ consisting of functions $\phi$ satisfying $D^{\alpha} \hat{\phi}(0)=0$ for all $\alpha \in \mathbb{N}^{d}$ where $\hat{\text { is }}$ the Fourier transform on $\mathscr{S}$ and $\mathscr{S}_{0}^{\prime}$ its topological dual space. One can see $\mathscr{S}_{0}^{\prime}$ as $\mathscr{S}^{\prime} / \mathscr{P}$ where $\mathscr{P}$ is the set of all polynomials on $\mathbb{R}^{d}$. Under these settings, $H_{q}^{\gamma}$ and $\dot{H}_{q}^{\gamma}$ are Banach spaces with the norms $\|u\|_{H_{q}^{\gamma}}$ and $\|u\|_{\dot{H}_{q}^{\gamma}}$ respectively. In the sequel, we shall use $H^{\gamma}:=H_{2}^{\gamma}, \dot{H}^{\gamma}:=\dot{H}_{2}^{\gamma}$. We also have for $\gamma>0, H_{q}^{\gamma}=L^{q} \cap \dot{H}_{q}^{\gamma}$.

Throughout this note, a pair $(p, q)$ is said to be admissible if

$$
(p, q) \in[2, \infty]^{2}, \quad(p, q, d) \neq(2, \infty, 2), \quad \frac{2}{p}+\frac{d}{q} \leq \frac{d}{2} .
$$

We also denote for $(p, q) \in[1, \infty]^{2}$,

$$
\gamma_{p, q}=\frac{d}{2}-\frac{d}{q}-\frac{4}{p}
$$

Since we are working in spaces of fractional order $\gamma$ or $\beta$, we need the nonlinearity $F(z)=$ $-\mu|z|^{\nu-1} z$ to have enough regularity. When $\nu$ is an odd integer, $F \in C^{\infty}(\mathbb{C}, \mathbb{C}$ ) (in the real sense). When $\nu$ is not an odd integer, we need the following assumption

$$
\lceil\gamma\rceil \text { or }\lceil\beta\rceil \leq \nu
$$

where $\lceil\gamma\rceil$ is the smallest integer greater than or equal to $\gamma$, similarly for $\beta$. Our first result concerns the local well-posedness of (NL4S) in both sub-critical and critical cases. 
Theorem 1.1. Let $\gamma \in[0, d / 2)$ be such that $\gamma \geq \gamma_{\mathrm{c}}$, and also, if $\nu>1$ is not an odd integer, (1.4). Let

$$
p=\frac{8(\nu+1)}{(\nu-1)(d-2 \gamma)}, \quad q=\frac{d(\nu+1)}{d+(\nu-1) \gamma} .
$$

Then for all $u_{0} \in H^{\gamma}$, there exist $T^{*} \in(0, \infty]$ and a unique solution to (NL4S) satisfying

$$
u \in C\left(\left[0, T^{*}\right), H^{\gamma}\right) \cap L_{\mathrm{loc}}^{p}\left(\left[0, T^{*}\right), H_{q}^{\gamma}\right) .
$$

Moreover, the following properties hold:

(i) $u \in L_{\text {loc }}^{a}\left(\left[0, T^{*}\right), H_{b}^{\gamma}\right)$ for any admissible pair $(a, b)$ with $b<\infty$ and $\gamma_{a, b}=0$.

(ii) $M(u(t))=M\left(u_{0}\right)$ for any $t \in\left[0, T^{*}\right)$.

(iii) If $\gamma \geq 2, E(u(t))=E\left(u_{0}\right)$ for any $t \in\left[0, T^{*}\right)$.

(iv) If $\gamma>\gamma_{\mathrm{c}}$ and $T^{*}<\infty$, then $\|u(t)\|_{\dot{H}^{\gamma}} \rightarrow \infty$ as $t \rightarrow T^{*}$.

(v) If $\gamma=\gamma_{\mathrm{c}}$ and $T^{*}<\infty$, then $\|u\|_{L^{p}\left(\left[0, T^{*}\right), H_{q}^{\gamma^{\mathrm{c}}}\right)}=\infty$.

(vi) $u$ depends continuously on $u_{0}$ in the following sense. There exists $0<T<T^{*}$ such that if $u_{0, n} \rightarrow u_{0}$ in $H^{\gamma}$ and if $u_{n}$ denotes the solution of (NL4S) with initial data $u_{0, n}$, then $0<T<T^{*}\left(u_{0, n}\right)$ for all $n$ sufficiently large and $u_{n}$ is bounded in $L^{a}\left([0, T], H_{b}^{\gamma}\right)$ for any admissible pair $(a, b)$ with $\gamma_{a, b}=0$ and $b<\infty$. Moreover, $u_{n} \rightarrow u$ in $L^{a}\left([0, T], L^{b}\right)$ as $n \rightarrow \infty$. In particular, $u_{n} \rightarrow u$ in $C\left([0, T], H^{\gamma-\epsilon}\right)$ for all $\epsilon>0$.

(vii) If $\gamma=\gamma_{\mathrm{c}}$ and $\left\|u_{0}\right\|_{\dot{H} \gamma_{c}}<\varepsilon$ for some $\varepsilon>0$ small enough, then $T^{*}=\infty$ and the solution is scattering in $H^{\gamma_{c}}$, i.e. there exists $u_{0}^{+} \in H^{\gamma_{c}}$ such that

$$
\lim _{t \rightarrow+\infty}\left\|u(t)-e^{i t \Delta^{2}} u_{0}^{+}\right\|_{H^{\gamma_{c}}}=0
$$

We also have the following local well-posedness in the critical Sobolev space $H^{d / 2}$.

Theorem 1.2. Let $\gamma=d / 2$ be such that if $\nu>1$ is not an odd integer, (1.4). Then for all $u_{0} \in H^{d / 2}$, there exists $T^{*} \in(0, \infty]$ and a unique solution to (NL4S) satisfying

$$
u \in C\left(\left[0, T^{*}\right), H^{d / 2}\right) \cap L_{\mathrm{loc}}^{p}\left(\left[0, T^{*}\right), L^{\infty}\right),
$$

for some $p>\max (\nu-1,4)$ when $d=1$ and some $p>\max (\nu-1,2)$ when $d \geq 2$. Moreover, the following properties hold:

(i) $u \in L_{\mathrm{loc}}^{a}\left(\left[0, T^{*}\right), H_{b}^{d / 2}\right)$ for any admissible pair $(a, b)$ with $b<\infty$ and $\gamma_{a, b}=0$.

(ii) If $T^{*}<\infty$, then $\|u(t)\|_{H^{d / 2}} \rightarrow \infty$ as $t \rightarrow T^{*}$.

(iii) $u$ depends continuously on $u_{0}$ in the sense of Theorem 1.1

The continuous dependence can be improved (see Remark 2.7) if we assume that $\nu>1$ is an odd integer or $\lceil d / 2\rceil \leq \nu-1$. Concerning the well-posedness of the nonlinear Schrödinger equation in this critical space, we refer to [22] and 25]. Note that in [25], the global wellposedness with small data is proved with exponential-type nonlinearity but not the local wellposedness without size restriction on the initial data.

It is well-known that (see Chapter 4 of [5], [22] or Chapter 3 of [30]) that for $\gamma>d / 2$, the nonlinear Schrödinger equation is locally well-posed provided the nonlinearity has enough regularity. It is not a problem to extend this result for the nonlinear fourth-order Schrödinger equation. For the sake of completeness, we state (without proof) the local well-posedness for (NL4S) in this range. 
Theorem 1.3. Let $\gamma>d / 2$ be such that if $\nu>1$ is not an odd integer, (1.4). Then for all $u_{0} \in H^{\gamma}$, there exist $T^{*} \in(0, \infty]$ and a unique solution $u \in C\left(\left[0, T^{*}\right), H^{\gamma}\right)$ to (NL4S). Moreover, the following properties hold:

(i) $u \in L_{\text {loc }}^{a}\left(\left[0, T^{*}\right), H_{b}^{\gamma}\right)$ for any admissible pair $(a, b)$ with $b<\infty$ and $\gamma_{a, b}=0$.

(ii) If $T^{*}<\infty$, then $\|u(t)\|_{H^{\gamma}} \rightarrow \infty$ and limsup $\|u(t)\|_{L^{\infty}} \rightarrow \infty$ as $t \rightarrow T^{*}$.

(iii) $u$ depends continuously on $u_{0}$ in the following sense. There exists $0<T<T^{*}$ such that if $u_{0, n} \rightarrow u_{0}$ in $H^{\gamma}$ and if $u_{n}$ is the solution of (NL4S) with the initial data $u_{0, n}$, then $u_{n} \rightarrow u$ in $C\left([0, T], H^{\gamma}\right)$.

Corollary 1.4. Let $\nu \in(1,1+8 / d)$. Then for all $\varphi \in L^{2}$, there exists a unique global solution to (NL4S) satisfying $u \in C\left(\mathbb{R}, L^{2}\right) \cap L_{\mathrm{loc}}^{p}\left(\mathbb{R}, L^{q}\right)$, where $(p, q)$ given in (1.5).

In the energy space $H^{2}$, we have the following global well-posedness result.

Proposition 1.5 (26] or [9]). Let $\nu \in(1,1+8 /(d-4))$ for $d \geq 5$ and $\nu>1$ for $d \leq 4$. Then for any $u_{0} \in H^{2}$, the solution to (NL4S) given in Theorem 1.1. Theorem 1.2 and Theorem 1.3 can be extended to the whole $\mathbb{R}$ if one of the following is satisfied:

(i) $\mu=1$.

(ii) $\mu=-1, \nu<1+8 / d$.

(iii) $\mu=-1, \nu=1+8 / d$ and $\left\|u_{0}\right\|_{L^{2}}$ is small.

(iv) $\mu=-1$ and $\left\|u_{0}\right\|_{H^{2}}$ is small.

Our next result concerns with the regularity of solutions of (NL4S) in the sub-critical case.

Theorem 1.6. Let $\beta>\gamma \geq 0$ be such that $\gamma>\gamma_{\mathrm{c}}$, and also, if $\nu>1$ is not an odd integer, (1.4). Let $u_{0} \in H^{\gamma}$ and $u$ be the corresponding $H^{\gamma}$ solution of (NL4S) given in Theorem 1.1. Theorem 1.2, Theorem 1.3. If $u_{0} \in H^{\beta}$, then $u \in C\left(\left[0, T^{*}\right), H^{\beta}\right)$.

The following result is a direct consequence of Theorem 1.6 and the global well-posedness in Corollary 1.4 and Proposition 1.5

Corollary 1.7. (i) Let $\gamma \geq 0$ and $\nu \in(1,1+8 / d)$ be such that if $\nu$ is not an odd integer, (1.4). Then (NL4S) is globally well-posed in $H^{\gamma}$.

(ii) Let $\gamma \geq 2, \nu \in[1+8 / d, 1+8 /(d-4))$ for $d \geq 5$ and $\nu \in[1+8 / d, \infty)$ for $d \leq 4$ be such that if $\nu$ is not an odd integer, (1.4). Then (NL4S) is globally well-posed in $H^{\gamma}$ provided one of conditions (i), (iii), (iv) in Proposition 1.5 is satisfied.

Our final result is the following ill-posedness for (NL4S).

Theorem 1.8. Let $\nu>1$ be such that if $\nu$ is not an odd integer, $\nu \geq k+1$ for some integer $k>d / 2$. Then (NL4S) is ill-posed in $H^{\gamma}$ for $\gamma \in\left((-\infty,-d / 2] \cap\left(-\infty, \gamma_{\mathrm{c}}\right)\right) \cup\left[0, \gamma_{\mathrm{c}}\right)$. Precisely, if $\gamma \in\left((-\infty,-d / 2] \cap\left(-\infty, \gamma_{\mathrm{c}}\right)\right) \cup\left(0, \gamma_{\mathrm{c}}\right)$, then for any $t>0$ the solution map $\mathscr{S} \ni u(0) \mapsto u(t)$ of (NL4S) fails to be continuous at 0 in the $H^{\gamma}$ topology. Moreover, if $\gamma_{\mathrm{c}}>0$, the solution map fails to be uniformly continuous on $L^{2}$.

The proof of Theorem 1.8 bases on the small dispersion analysis given in [7]. Note that when $\nu=3$ and $\mu=1$ corresponding to the defocusing cubic nonlinearity, Pausader in [27] proves the ill-posedness for (NL4S) in $H^{2}\left(\mathbb{R}^{d}\right)$ with $d \geq 9$.

This note is organized as follows. In Section 2 we recall Strichartz estimate for the linear fourth-order Schrödinger equation and the nonlinear fractional derivatives. We end this section with the proof the local well-posedness given in Theorem 1.1 and Theorem 1.2. In Section 3, we give the proof of the regularity for solutions of (NL4S) given in Theorem 1.6. Finally, the proof of the ill-posedness result is given in Section 4 . 


\section{Well-posedness}

In this section, we will give the proofs of the local well-posedness given in Theorem 1.1 and Theorem 1.2. Our proofs are based on the standard contraction mapping argument using Strichartz estimate and nonlinear fractional derivatives (see Subsection 2.2).

\section{$2.1 \quad$ Strichartz estimate}

In this subsection, we recall Strichartz estimate for the fourth-order Schrödinger equation.

Proposition 2.1 ([9]). Let $\gamma \in \mathbb{R}$ and u be a (weak) solution to the linear fourth-order Schrödinger equation, namely

$$
u(t)=e^{i t \Delta^{2}} u_{0}+\int_{0}^{t} e^{i(t-s) \Delta^{2}} F(s) d s,
$$

for some data $u_{0}, F$. Then for all $(p, q)$ and $(a, b)$ admissible with $q<\infty$ and $b<\infty$,

$$
\|u\|_{L^{p}\left(\mathbb{R}, L^{q}\right)} \lesssim\left\|u_{0}\right\|_{\dot{H}^{\gamma_{p}, q}}+\|F\|_{L^{a^{\prime}\left(\mathbb{R}, L^{b^{\prime}}\right)}}
$$

provided that

$$
\gamma_{p, q}=\gamma_{a^{\prime}, b^{\prime}}+4
$$

Here $\left(a, a^{\prime}\right)$ is a conjugate pair and similarly for $\left(b, b^{\prime}\right)$.

Remark 2.2. The estimate (2.1) is exactly the one given in 26] or 27] where the author considered $(p, q)$ and $(a, b)$ are Schrödinger admissible, i.e.

$$
p, q \in[2, \infty]^{2}, \quad(p, q, d) \neq(2, \infty, 2), \quad \frac{2}{p}+\frac{d}{q}=\frac{d}{2} .
$$

We refer to 9 for the proof of Proposition 2.1. Note that rather than using directly a dedicate dispersive estimate of [1] for the fundamental solution of the homogeneous fourth-order Schrödinger equation, we use scaling technique which is similar to those of wave equation (see e.g. [23]). Our Strichartz estimate is flexible enough to show the local well-posedness for (NL4S) in both sub-critical and critical cases.

We also have the following local Strichartz estimate (see again [9]).

Corollary 2.3. Let $\gamma \geq 0$ and $I$ be a bounded interval. If $u$ is a weak solution to the linear fourth-order Schrödinger equation for some data $u_{0}, F$, then for all $(p, q)$ admissible satisfying $q<\infty$

$$
\|u\|_{L^{p}\left(I, H_{q}^{\left.\gamma-\gamma_{p, q}\right)}\right.} \lesssim\left\|u_{0}\right\|_{H^{\gamma}}+\|F\|_{L^{1}\left(I, H^{\gamma}\right)}
$$

\subsection{Nonlinear fractional derivatives}

In this subsection, we recall some nonlinear fractional derivatives estimates related to our purpose. Let us start with the following fractional Leibniz rule (or Kato-Ponce inequality). We refer to [15] for the proof of a more general result.

Proposition 2.4. Let $\gamma \geq 0,1<r<\infty$ and $1<p_{1}, p_{2}, q_{1}, q_{2} \leq \infty$ satisfying

$$
\frac{1}{r}=\frac{1}{p_{1}}+\frac{1}{q_{1}}=\frac{1}{p_{2}}+\frac{1}{q_{2}} .
$$

Then there exists $C=C\left(d, \gamma, r, p_{1}, q_{1}, p_{2}, q_{2}\right)>0$ such that for all $u, v \in \mathscr{S}$,

$$
\left\|\Lambda^{\gamma}(u v)\right\|_{L^{r}} \leq C\left(\left\|\Lambda^{\gamma} u\right\|_{L^{p_{1}}}\|v\|_{L^{q_{1}}}+\|u\|_{L^{p_{2}}}\left\|\Lambda^{\gamma} v\right\|_{L^{q_{2}}}\right)
$$


We also have the following fractional chain rule (see [8] or 29]).

Proposition 2.5. Let $F \in C^{1}(\mathbb{C}, \mathbb{C})$ and $G \in C\left(\mathbb{C}, \mathbb{R}^{+}\right)$such that $F(0)=0$ and

$$
\left|F^{\prime}(\theta z+(1-\theta) \zeta)\right| \leq \mu(\theta)(G(z)+G(\zeta)), \quad z, \zeta \in \mathbb{C}, \quad 0 \leq \theta \leq 1,
$$

where $\mu \in L^{1}((0,1))$. Then for $\gamma \in(0,1)$ and $1<r, p<\infty, 1<q \leq \infty$ satisfying

$$
\frac{1}{r}=\frac{1}{p}+\frac{1}{q}
$$

there exists $C=C(d, \mu, \gamma, r, p, q)>0$ such that for all $u \in \mathscr{S}$,

$$
\left\|\Lambda^{\gamma} F(u)\right\|_{L^{r}} \leq C\left\|F^{\prime}(u)\right\|_{L^{q}}\left\|\Lambda^{\gamma} u\right\|_{L^{p}} .
$$

Combining the fractional Leibniz rule and the fractional chain rule, one has the following result (see the appendix of [22]).

Lemma 2.6. Let $F \in C^{k}(\mathbb{C}, \mathbb{C}), k \in \mathbb{N} \backslash\{0\}$. Assume that there is $\nu \geq k$ such that

$$
\left|D^{i} F(z)\right| \leq C|z|^{\nu-i}, \quad z \in \mathbb{C}, \quad i=1,2, \ldots, k .
$$

Then for $\gamma \in[0, k]$ and $1<r, p<\infty, 1<q \leq \infty$ satisfying $\frac{1}{r}=\frac{1}{p}+\frac{\nu-1}{q}$, there exists $C=C(d, \nu, \gamma, r, p, q)>0$ such that for all $u \in \mathscr{S}$,

$$
\left\|\Lambda^{\gamma} F(u)\right\|_{L^{r}} \leq C\|u\|_{L^{q}}^{\nu-1}\left\|\Lambda^{\gamma} u\right\|_{L^{p}} .
$$

Moreover, if $F$ is a homogeneous polynomial in $u$ and $\bar{u}$, then (2.4) holds true for any $\gamma \geq 0$.

\subsection{Proof of Theorem 1.1}

We are now able to prove Theorem 1.1. Let us firstly comment about the choice of $(p, q)$ given in (1.5). It is easy to see that $(p, q)$ is admissible and $\gamma_{p, q}=0=\gamma_{p^{\prime}, q^{\prime}}+4$. This allows us to use Strichartz estimate (2.1) for $(p, q)$. Moreover, if we choose $(m, n)$ so that

$$
\frac{1}{p^{\prime}}=\frac{1}{m}+\frac{\nu-1}{p}, \quad \frac{1}{q^{\prime}}=\frac{1}{q}+\frac{\nu-1}{n},
$$

Thanks to this choice of $n$, we have the Sobolev embedding $\dot{H}_{q}^{\gamma} \hookrightarrow L^{n}$ since

$$
q \leq n=\frac{d q}{d-\gamma q}
$$

Step 1. Existence. Let us consider

$$
X:=\left\{u \in L^{p}\left(I, H_{q}^{\gamma}\right) \mid\|u\|_{L^{p}\left(I, \dot{H}_{q}^{\gamma}\right)} \leq M\right\},
$$

equipped with the distance

$$
d(u, v)=\|u-v\|_{L^{p}\left(I, L^{q}\right)},
$$

where $I=[0, T]$ and $M, T>0$ to be chosen later. It is easy to verify (see e.g. 4] or Chapter 4 of [5]) that $(X, d)$ is a complete metric space. By the Duhamel formula, it suffices to prove that the functional

$$
\Phi(u)(t)=e^{i t \Delta^{2}} u_{0}+i \mu \int_{0}^{t} e^{i(t-s) \Delta^{2}}|u(s)|^{\nu-1} u(s) d s=: u_{\mathrm{hom}}(t)+u_{\mathrm{inh}}(t)
$$

is a contraction on $(X, d)$.

Let us firstly consider the case $\gamma>\gamma_{c}$. In this case, we have $1<m<p$ and

$$
\frac{1}{m}-\frac{1}{p}=1-\frac{(\nu-1)(d-2 \gamma)}{8}=: \theta>0
$$


Using Strichartz estimate (2.1), we obtain

$$
\begin{aligned}
&\|\Phi(u)\|_{L^{p}\left(I, \dot{H}_{q}^{\gamma}\right)} \lesssim\left\|u_{0}\right\|_{\dot{H}^{\gamma}}+\|F(u)\|_{L^{p^{\prime}\left(I, \dot{H}_{q^{\prime}}^{\gamma}\right)}}, \\
&\|\Phi(u)-\Phi(v)\|_{L^{p}\left(I, L^{q}\right)} \lesssim\|F(u)-F(v)\|_{L^{p^{\prime}\left(I, L^{q^{\prime}}\right)}},
\end{aligned}
$$

where $F(u)=|u|^{\nu-1} u$ and similarly for $F(v)$. It then follows from Lemma 2.6. [2.5), Sobolev embedding and (2.7) that

$$
\begin{aligned}
\|F(u)\|_{L^{p^{\prime}\left(I, \dot{H}_{q^{\prime}}^{\gamma}\right)}} & \lesssim T^{\theta}\|u\|_{L^{p}\left(I, \dot{H}_{q}^{\gamma}\right)}^{\nu} \\
\|F(u)-F(v)\|_{L^{p^{\prime}\left(I, L^{q^{\prime}}\right)}} & \lesssim T^{\theta}\left(\|u\|_{L^{p}\left(I, \dot{H}_{q}^{\gamma}\right)}^{\nu-1}+\|v\|_{L^{p}\left(I, \dot{H}_{q}^{\gamma}\right)}^{\nu-1}\right)\|u-v\|_{L^{p}\left(I, L^{q}\right)} .
\end{aligned}
$$

This shows that for all $u, v \in X$, there exists $C>0$ independent of $T$ and $u_{0} \in H^{\gamma}$ such that

$$
\begin{gathered}
\|\Phi(u)\|_{L^{p}\left(I, \dot{H}_{q}^{\gamma}\right)} \leq C\left\|u_{0}\right\|_{\dot{H}^{\gamma}}+C T^{\theta} M^{\nu}, \\
d(\Phi(u), \Phi(v)) \leq C T^{\theta} M^{\nu-1} d(u, v) .
\end{gathered}
$$

If we set $M=2 C\left\|u_{0}\right\|_{\dot{H}^{\gamma}}$ and choose $T>0$ so that

$$
C T^{\theta} M^{\nu-1} \leq \frac{1}{2}
$$

then $\Phi$ is a strict contraction on $(X, d)$.

We now turn to the case $\gamma=\gamma_{\mathrm{c}}$. We have from Strichartz estimate (2.1) that

$$
\left\|u_{\text {hom }}\right\|_{L^{p}\left(I, \dot{H}_{q}^{\gamma_{c}}\right)} \lesssim\left\|u_{0}\right\|_{\dot{H}^{\gamma_{c}}} \text {. }
$$

This shows that $\left\|u_{\text {hom }}\right\|_{L^{p}\left(I, \dot{H}_{q}^{\left.\gamma_{c}\right)}\right.} \leq \varepsilon$ for some $\varepsilon>0$ small enough provided that $T$ is small or $\left\|u_{0}\right\|_{\dot{H}^{\gamma_{c}}}$ is small. We also have from (2.1) that

$$
\left\|u_{\mathrm{inh}}\right\|_{L^{p}\left(I, \dot{H}_{q}^{\gamma_{c}}\right)} \lesssim\|F(u)\|_{L^{p^{\prime}\left(I, \dot{H}_{q^{\prime}}^{\gamma_{\mathrm{c}}}\right.}} .
$$

Lemma (2.6), (2.5) and Sobolev embedding (note that in this case $m=p$ ) then yield that

$$
\begin{aligned}
&\|F(u)\|_{L^{p^{\prime}\left(I, \dot{H}_{q^{\prime}}^{\gamma_{\mathrm{c}}}\right)}} \lesssim\|u\|_{L^{p}\left(I, \dot{H}_{q}^{\gamma_{\mathrm{c}}}\right)}^{\nu} \\
&\|F(u)-F(v)\|_{L^{p^{\prime}\left(I, L^{q^{\prime}}\right)}} \lesssim\left(\|u\|_{L^{p}\left(I, \dot{H}_{q}^{\gamma_{c}}\right)}^{\nu}+\|v\|_{L^{p}\left(I, \dot{H}_{q}^{\gamma_{c}}\right)}^{\nu-1}\right)\|u-v\|_{L^{p}\left(I, L^{q}\right)} .
\end{aligned}
$$

This implies that for all $u, v \in X$, there exists $C>0$ independent of $T$ and $u_{0} \in H^{\gamma_{\mathrm{c}}}$ such that

$$
\begin{aligned}
\|\Phi(u)\|_{L^{p}\left(I, \dot{H}_{q}^{\gamma^{c}}\right)} & \leq \varepsilon+C M^{\nu}, \\
d(\Phi(u), \Phi(v)) & \leq C M^{\nu-1} d(u, v) .
\end{aligned}
$$

If we choose $\varepsilon$ and $M$ small so that

$$
C M^{\nu-1} \leq \frac{1}{2}, \quad \varepsilon+\frac{M}{2} \leq M,
$$

then $\Phi$ is a contraction on $(X, d)$.

Therefore, in both sub-critical and critical cases, $\Phi$ has a unique fixed point in $X$. Moreover, since $u_{0} \in H^{\gamma}$ and $u \in L^{p}\left(I, H_{q}^{\gamma}\right)$, the Strichartz estimate shows that $u \in C\left(I, H^{\gamma}\right)$ (see e.g. 4] or Chapter 4 of [5). This shows the existence of solution $u \in C\left(I, H^{\gamma}\right) \cap L^{p}\left(I, H_{q}^{\gamma}\right)$ to (NL4S). Note that in the case $\gamma=\gamma_{\mathrm{c}}$, if $\left\|u_{0}\right\|_{\dot{H}^{\gamma_{\mathrm{c}}}}$ is small enough, then we can take $T=\infty$.

Step 2. Uniqueness. It follows easily from (2.9) and (2.11) using the fact that $\|u\|_{L^{p}\left(I, \dot{H}_{q}^{\gamma}\right)}$ can be small if $T$ is small.

Step 3. Item (i). Let $u \in C\left(I, H^{\gamma}\right) \cap L^{p}\left(I, H_{q}^{\gamma}\right)$ be a solution to (NLFS) where $I=[0, T]$ and $(a, b)$ an admissible pair with $b<\infty$ and $\gamma_{a, b}=0$. Then Strichartz estimate (2.1) implies

$$
\begin{aligned}
\|u\|_{L^{a}\left(I, L^{b}\right)} & \lesssim\left\|u_{0}\right\|_{L^{2}}+\|F(u)\|_{L^{p^{\prime}}\left(I, L^{q^{\prime}}\right)}, \\
\|u\|_{L^{a}\left(I, \dot{H}_{b}^{\gamma}\right)} & \lesssim\left\|u_{0}\right\|_{\dot{H}^{\gamma}}+\|F(u)\|_{L^{p^{\prime}\left(I, \dot{H}_{q^{\prime}}^{\gamma}\right.}} .
\end{aligned}
$$


It then follows from (2.8) and (2.10) that $u \in L^{a}\left(I, H_{b}^{\gamma}\right)$.

Step 4. Item (ii) and (iii). The conservation of mass and energy follows similarly as for the Schrödinger equation (see e.g. 4], Chapter 4 of [5] or Chapter 5 of [13]).

Step 5. Item (iv). The blowup alternative in sub-critical case is easy since the time of existence depends only on $\left\|u_{0}\right\|_{\dot{H}^{\gamma}}$.

Step 6. Item (v). It also follows from a standard argument (see e.g. [4). Indeed, if $T^{*}<\infty$ and $\|u\|_{L^{p}\left(\left[0, T^{*}\right), H_{q}^{\gamma_{c}}\right)}<\infty$, then Strichartz estimate (2.1) implies that $u \in C\left(\left[0, T^{*}\right], H^{\gamma_{c}}\right)$. Thus, one can extend the solution to (NL4S) beyond $T^{*}$. It leads to a contradiction with the maximality of $T^{*}$.

Step 7. Item (vi). We use the argument given in [4. From Step 1, in the sub-critical case, we can choose $T$ and $M$ so that the fixed point argument can be carried out on $X$ for any initial data with $\dot{H}^{\gamma}$ norm less than $2\left\|u_{0}\right\|_{\dot{H}^{\gamma}}$. In the critical case, there exist $T, M$ and an $\dot{H}^{\gamma_{c}}$ neighborhood $U$ of $u_{0}$ such that the fixed point argument can be carried out on $X$ for all initial data in $U$. Now let $u_{0, n} \rightarrow u_{0}$ in $H^{\gamma}$. In both sub-critical and critical cases, we see that $T<T^{*}\left(u_{0}\right)$, $\|u\|_{L^{p}\left([0, T], \dot{H}_{q}^{\gamma}\right)} \leq M$, and that for sufficiently large $n, T<T^{*}\left(u_{0, n}\right)$ and $\left\|u_{n}\right\|_{L^{p}\left([0, T], \dot{H}_{q}^{\gamma}\right)} \leq M$. Thus, (2.12) and (2.13) together with (2.8) and (2.10) yield that $u_{n}$ is bounded in $L^{a}\left([0, T], H_{b}^{\gamma}\right)$ for any admissible pair $(a, b)$ with $b<\infty$ and $\gamma_{a, b}=0$. We also have from (2.9), (2.11) and the choice of $T$ that

$$
d\left(u_{n}, u\right) \leq C\left\|u_{0, n}-u_{0}\right\|_{L^{2}}+\frac{1}{2} d\left(u_{n}, u\right) \text { or } d\left(u_{n}, u\right) \leq 2 C\left\|u_{0, n}-u_{0}\right\|_{L^{2}} .
$$

This shows that $u_{n} \rightarrow u$ in $L^{p}\left([0, T], L^{q}\right)$. Again (2.13) together with (2.9) and (2.11) implies that $u_{n} \rightarrow u$ in $L^{a}\left([0, T], L^{b}\right)$ for any admissible pair $(a, b)$ with $b<\infty$ and $\gamma_{a, b}=0$. The convergence in $C\left(I, H^{\gamma-\epsilon}\right)$ follows from the boundedness in $L^{\infty}\left(I, H^{\gamma}\right)$ and the convergence in $L^{\infty}\left(I, L^{2}\right)$ and that $\|u\|_{H^{\gamma-\epsilon}} \leq\|u\|_{H^{\gamma}}^{1-\frac{\epsilon}{\gamma}}\|u\|_{L^{2}}^{\frac{\epsilon}{\gamma}}$.

Step 8. Item (vii). As mentioned in Step 1, when $\left\|u_{0}\right\|_{\dot{H}^{\gamma_{c}}}$ is small, we can take $T^{*}=\infty$. It remains to prove the scattering property. To do so, we make use of the adjoint estimate to the homogeneous Strichartz estimate, namely $L^{2} \ni u_{0} \mapsto e^{i t \Delta^{2}} u_{0} \in L^{p}\left(\mathbb{R}, L^{q}\right)$ to obtain

$$
\begin{aligned}
\left\|e^{-i t_{2} \Delta^{2}} u\left(t_{2}\right)-e^{-i t_{1} \Delta^{2}} u\left(t_{1}\right)\right\|_{\dot{H} \gamma_{c}} & =\left\|i \mu \int_{t_{1}}^{t_{2}} e^{-i s \Delta^{2}} F(u)(s) d s\right\|_{\dot{H}^{\gamma_{c}}} \\
& =\left\|i \mu \int_{t_{1}}^{t_{2}} \Lambda^{\gamma_{c}} e^{-i s \Delta^{2}}\left(\mathbb{1}_{\left[t_{1}, t_{2}\right]} F(u)\right)(s) d s\right\|_{L^{2}} \\
& \lesssim\|F(u)\|_{L^{p^{\prime}}\left(\left[t_{1}, t_{2}\right], \dot{H}_{q^{\prime}}^{\gamma_{c}}\right)} .
\end{aligned}
$$

Similarly,

$$
\left\|e^{-i t_{2} \Delta^{2}} u\left(t_{2}\right)-e^{-i t_{1} \Delta^{2}} u\left(t_{1}\right)\right\|_{L^{2}} \lesssim\|F(u)\|_{L^{p^{\prime}}\left(\left[t_{1}, t_{2}\right], L^{q^{\prime}}\right)} .
$$

Thanks to (2.10) and (2.11), we get

$$
\left\|e^{-i t_{2} \Delta^{2}} u\left(t_{2}\right)-e^{-i t_{1} \Delta^{2}} u\left(t_{1}\right)\right\|_{H \gamma_{c}} \rightarrow 0,
$$

as $t_{1}, t_{2} \rightarrow+\infty$. This implies that the limit

$$
u_{0}^{+}:=\lim _{t \rightarrow+\infty} e^{-i t \Delta^{2}} u(t)
$$

exists in $H^{\gamma_{c}}$. Moreover,

$$
u(t)-e^{i t \Delta^{2}} u_{0}^{+}=-i \mu \int_{t}^{+\infty} e^{i(t-s) \Delta^{2}} F(u(s)) d s .
$$

Using again (2.14) and (2.15) together with (2.10) and (2.11), we have

$$
\lim _{t \rightarrow+\infty}\left\|u(t)-e^{i t \Delta^{2}} u_{0}^{+}\right\|_{H \gamma_{c}}=0 .
$$

This completes the proof of Theorem 1.1 


\subsection{Proof of Theorem 1.2}

We now turn to the proof of the local well-posedness in $H^{d / 2}$. To do so, we firstly choose $p>\max (\nu-1,4)$ when $d=1$ and $p>\max (\nu-1,2)$ when $d \geq 2$ and then choose $q \in[2, \infty)$ such that

$$
\frac{2}{p}+\frac{d}{q} \leq \frac{d}{2}
$$

Step 1. Existence. We will show that $\Phi$ defined in (2.6) is a contraction on

$$
X:=\left\{u \in L^{\infty}\left(I, H^{d / 2}\right) \cap L^{p}\left(I, H_{q}^{d / 2-\gamma_{p, q}}\right) \mid\|u\|_{L^{\infty}\left(I, H^{d / 2}\right)}+\|u\|_{L^{p}\left(I, H_{q}^{d / 2-\gamma_{p}, q}\right)} \leq M\right\},
$$

equipped with the distance

$$
d(u, v):=\|u-v\|_{L^{\infty}\left(I, L^{2}\right)}+\|u-v\|_{L^{p}\left(I, H^{-\gamma p, q}\right)},
$$

where $I=[0, T]$ and $M, T>0$ to be determined. The local Strichartz estimate (2.3) gives

$$
\begin{aligned}
\|\Phi(u)\|_{L^{\infty}\left(I, H^{d / 2}\right)}+\|\Phi(u)\|_{L^{p}\left(I, H_{q}^{d / 2-\gamma_{p}, q}\right)} & \lesssim\left\|u_{0}\right\|_{H^{d / 2}}+\|F(u)\|_{L^{1}\left(I, H^{d / 2}\right)}, \\
\|\Phi(u)-\Phi(v)\|_{L^{\infty}\left(I, L^{2}\right)}+\|\Phi(u)-\Phi(v)\|_{L^{p}\left(I, H_{q}^{-\gamma_{p}, q}\right)} & \lesssim\|F(u)-F(v)\|_{L^{1}\left(I, L^{2}\right)} .
\end{aligned}
$$

Thanks to the assumptions on $\nu$, Lemma 2.6 implies

$$
\begin{aligned}
\|F(u)\|_{L^{1}\left(I, H^{d / 2}\right)} & \lesssim\|u\|_{L^{\nu-1}\left(I, L^{\infty}\right)}^{\nu-1}\|u\|_{L^{\infty}\left(I, H^{d / 2}\right)} \lesssim T^{\theta}\|u\|_{L^{p}\left(I, L^{\infty}\right)}^{\nu-1}\|u\|_{L^{\infty}\left(I, H^{d / 2}\right)}, \\
\|F(u)-F(v)\|_{L^{1}\left(I, L^{2}\right)} & \lesssim\left(\|u\|_{L^{\nu-1}\left(I, L^{\infty}\right)}^{\nu-1}+\|v\|_{L^{\nu-1}\left(I, L^{\infty}\right)}^{\nu-1}\right)\|u-v\|_{L^{\infty}\left(I, L^{2}\right)} \\
& \lesssim T^{\theta}\left(\|u\|_{L^{p}\left(I, L^{\infty}\right)}^{\nu-1}+\|v\|_{L^{p}\left(I, L^{\infty}\right)}^{\nu-1}\right)\|u-v\|_{L^{\infty}\left(I, L^{2}\right)},
\end{aligned}
$$

where $\theta=1-\frac{\nu-1}{p}>0$. Using the fact that $d / 2-\gamma_{p, q}>d / q$, the Sobolev embedding implies $H_{q}^{d / 2-\gamma_{p, q}} \hookrightarrow L^{\infty}$. Thus,

$$
\begin{aligned}
\|\Phi(u)\|_{L^{\infty}\left(I, H^{d / 2}\right)}+\|\Phi(v)\|_{L^{p}\left(I, H_{q}^{d / 2-\gamma_{p}, q}\right)} & \lesssim\left\|u_{0}\right\|_{H^{d / 2}}+T^{\theta}\|u\|_{L^{p}\left(I, H_{q}^{d / 2-\gamma_{p}, q}\right)}^{\nu-1}\|u\|_{L^{\infty}\left(I, H^{d / 2}\right)}, \\
d(\Phi(u), \Phi(v)) & \lesssim T^{\theta}\left(\|u\|_{L^{p}\left(I, H_{q}^{d / 2-\gamma_{p}, q}\right)}^{\nu-1}+\|v\|_{L^{p}\left(I, H_{q}^{d / 2-\gamma_{p}, q}\right)}^{\nu-1}\right) d(u, v) .
\end{aligned}
$$

Thus for all $u, v \in X$, there exists $C>0$ independent of $u_{0} \in H^{d / 2}$ such that

$$
\begin{aligned}
\|\Phi(u)\|_{L^{\infty}\left(I, H^{d / 2}\right)}+\|\Phi(v)\|_{L^{p}\left(I, H_{q}^{d / 2-\gamma_{p}, q}\right)} & \leq C\left\|u_{0}\right\|_{H^{d / 2}}+C T^{\theta} M^{\nu}, \\
d(\Phi(u), \Phi(v)) & \leq C T^{\theta} M^{\nu-1} d(u, v) .
\end{aligned}
$$

If we set $M=2 C\left\|u_{0}\right\|_{H^{d / 2}}$ and choose $T>0$ small enough so that $C T^{\theta} M^{\nu-1} \leq \frac{1}{2}$, then $\Phi$ is a contraction on $X$.

Step 2. Uniqueness. It is easy using (2.17) since $\|u\|_{L^{p}\left(I, L^{\infty}\right)}$ is small if $T$ is small.

Step 3. Item (i). It follows easily from Step 1 and Strichartz estimate (2.3) that for any admissible pair $(a, b)$ with $b<\infty$ and $\gamma_{a, b}=0$,

$$
\|u\|_{L^{a}\left(I, H_{b}^{d / 2}\right)} \lesssim\left\|u_{0}\right\|_{H^{d / 2}}+\|F(u)\|_{L^{1}\left(I, H^{d / 2}\right)} .
$$

Step 4. Item (ii). The blowup alternative is obvious since the time of existence depends only on $\left\|u_{0}\right\|_{H^{d / 2}}$.

Step 5. Item (iii). The continuous dependence is similar to Step 7 of the proof of Theorem 1.1 using (2.17).

Remark 2.7. If we assume that $\nu>1$ is an odd integer or $\lceil d / 2\rceil \leq \nu-1$ otherwise, then the continuous dependence holds in $C\left(I, H^{d / 2}\right)$. Indeed, we consider $X$ as above equipped with the following metric

$$
d(u, v):=\|u-v\|_{L^{\infty}\left(I, H^{d / 2}\right)}+\|u-v\|_{L^{p}\left(I, H_{q}^{d / 2-\gamma_{p}, q}\right)} .
$$


Thanks to the assumptions on $\nu$, we are able to apply the fractional derivatives estimates (see e.g. the appendix of [22] or Corollary 3.5 of [9]) to have

$$
\begin{aligned}
\| F(u) & -F(v)\left\|_{L^{1}\left(I, H^{d / 2}\right)} \lesssim\left(\|u\|_{L^{\nu-1}\left(I, L^{\infty}\right)}^{\nu-1}+\|v\|_{L^{\nu-1}\left(I, L^{\infty}\right)}^{\nu-1}\right)\right\| u-v \|_{L^{\infty}\left(I, H^{d / 2}\right)} \\
& +\left(\|u\|_{L^{\nu-1}\left(I, L^{\infty}\right)}^{\nu-2}+\|v\|_{L^{\nu-1}\left(I, L^{\infty}\right)}^{\nu-2}\right)\left(\|u\|_{L^{\infty}\left(I, H^{d / 2}\right)}+\|v\|_{L^{\infty}\left(I, H^{d / 2}\right)}\right)\|u-v\|_{L^{\nu-1}\left(I, L^{\infty}\right)} .
\end{aligned}
$$

The Sobolev embedding then implies that for all $u, v \in X$,

$$
d(\Phi(u), \Phi(v)) \lesssim T^{\theta} M^{\nu-1} d(u, v) .
$$

The continuous dependence in $C\left(I, H^{d / 2}\right)$ follows as Step 7 of the proof of Theorem 1.1

\section{Regularity}

The main purpose of this section is to prove the regularity of solutions of (NL4S) given in Theorem 1.6. We follow the argument given in Chapter 5 of [5]. To do so, we will split $\gamma$ into three cases $\gamma \in[0, d / 2), \gamma=d / 2$ and $\gamma>d / 2$.

\subsection{The case $\gamma \in[0, d / 2)$}

Let $\beta>\gamma$. If $u_{0} \in H^{\beta}$, then Theorem 1.1 or Theorem 1.2 or Theorem 1.3 shows that there exists a maximal solution to (NL4S) satisfying $u \in C\left([0, T), H^{\beta}\right) \cap L_{\text {loc }}^{a}\left([0, T), H_{b}^{\beta}\right)$ for any admissible pair $(a, b)$ with $b<\infty$ and $\gamma_{a, b}=0$. Since $H^{\beta}$-solution is in particular an $H^{\gamma}$-solution, the uniqueness implies that $T \leq T^{*}$. We will show that $T$ is actually equal to $T^{*}$. Suppose that $T<T^{*}$, then the blowup alternative implies

$$
\|u(t)\|_{H^{\beta}} \rightarrow \infty \text { as } t \rightarrow T \text {. }
$$

Moreover, since $T<T^{*}$, we have

$$
\|u\|_{L^{p}\left((0, T), H_{q}^{\gamma}\right)}+\sup _{0 \leq t \leq T}\|u(t)\|_{H^{\gamma}}<\infty,
$$

where $(p, q)$ given in (1.5). Using Strichartz estimate (2.1), we have for any interval $I \subset(0, T)$,

$$
\begin{aligned}
\|u\|_{L^{\infty}\left(I, L^{2}\right)}+\|u\|_{L^{p}\left(I, L^{q}\right)} & \lesssim\left\|u_{0}\right\|_{L^{2}}+\|F(u)\|_{L^{p^{\prime}\left(I, L^{q^{\prime}}\right)}}, \\
\|u\|_{L^{\infty}\left(I, \dot{H}^{\beta}\right)}+\|u\|_{L^{p}\left(I, \dot{H}_{q}^{\beta}\right)} & \lesssim\left\|u_{0}\right\|_{\dot{H}^{\beta}}+\|F(u)\|_{L^{p^{\prime}\left(I, \dot{H}_{q^{\prime}}^{\beta}\right.}} .
\end{aligned}
$$

Now, let $(m, n)$ be as in (2.5). Lemma 2.6. (2.5) and Sobolev embedding then give

$$
\begin{aligned}
\|F(u)\|_{L^{p^{\prime}\left(I, L^{q^{\prime}}\right)}} \lesssim\|u\|_{L^{p}\left(I, L^{n}\right)}^{\nu-1}\|u\|_{L^{m}\left(I, L^{q}\right)} \lesssim\|u\|_{L^{p}\left(I, \dot{H}_{q}^{\gamma}\right)}^{\nu-1}\|u\|_{L^{m}\left(I, L^{q}\right)} \lesssim\|u\|_{L^{m}\left(I, L^{q}\right)}, \\
\|F(u)\|_{L^{p^{\prime}\left(I, \dot{H}_{q^{\prime}}^{\beta}\right)}} \lesssim\|u\|_{L^{p}\left(I, L^{n}\right)}^{\nu-1}\|u\|_{L^{m}\left(I, \dot{H}_{q}^{\beta}\right)} \lesssim\|u\|_{L^{p}\left(I, \dot{H}_{q}^{\gamma}\right)}^{\nu-1}\|u\|_{L^{m}\left(I, \dot{H}_{q}^{\beta}\right)} \lesssim\|u\|_{L^{m}\left(I, \dot{H}_{q}^{\beta}\right)} .
\end{aligned}
$$

Here we use the fact that $\|u\|_{L^{p}\left((0, T), H_{q}^{\gamma}\right)}$ is bounded. This shows that

$$
\|u\|_{L^{\infty}\left(I, H^{\beta}\right)}+\|u\|_{L^{p}\left(I, H_{q}^{\beta}\right)} \lesssim\left\|u_{0}\right\|_{H^{\beta}}+\|u\|_{L^{m}\left(I, H_{q}^{\beta}\right)},
$$

for every interval $I \subset(0, T)$. Now let $0<\epsilon<T$ and consider $I=(0, \tau)$ with $\epsilon<\tau<T$. We have

$$
\|u\|_{L^{m}\left(I, H_{q}^{\beta}\right)} \leq\|u\|_{L^{m}\left((0, \tau-\epsilon), H_{q}^{\beta}\right)}+\|u\|_{L^{m}\left((\tau-\epsilon, \tau), H_{q}^{\beta}\right)} \leq C_{\epsilon}+\epsilon^{\theta}\|u\|_{L^{p}\left(I, H_{q}^{\beta}\right)},
$$

where $\theta$ given in (2.7). Here we also use the fact that $u \in L_{\mathrm{loc}}^{p}\left([0, T), H_{q}^{\beta}\right)$ since $\gamma_{p, q}=0$. Thus,

$$
\|u\|_{L^{\infty}\left(I, H^{\beta}\right)}+\|u\|_{L^{p}\left(I, H_{q}^{\beta}\right)} \leq C+C_{\epsilon}+\epsilon^{\theta} C\|u\|_{L^{p}\left(I, H_{q}^{\beta}\right)},
$$

where the various constants are independent of $\tau<T$. By choosing $\epsilon$ small enough, we have

$$
\|u\|_{L^{\infty}\left(I, H^{\beta}\right)}+\|u\|_{L^{p}\left(I, H_{q}^{\beta}\right)} \leq C,
$$

where $C$ is independent of $\tau<T$. Let $\tau \rightarrow T$, we get a contradiction with (3.1). 


\subsection{The case $\gamma=d / 2$}

Since $u_{0} \in H^{d / 2}$, Theorem 1.2 shows that there exists a unique, maximal solution to (NL4S) satisfying $u \in C\left(\left[0, T^{*}\right), H^{d / 2}\right) \cap L_{\text {loc }}^{p}\left(\left[0, T^{*}\right), L^{\infty}\right)$ for some $p>\max (\nu-1,4)$ when $d=1$ and $p>\max (\nu-1,2)$ when $d \geq 2$. This implies in particular that

$$
u \in L_{\mathrm{loc}}^{\nu-1}\left(\left[0, T^{*}\right), L^{\infty}\right) .
$$

Now let $\beta>\gamma$. If $u_{0} \in H^{\beta}$, then we know that $u$ is an $H^{\beta}$ solution defined on some maximal interval $[0, T)$ with $T \leq T^{*}$. Suppose that $T<T^{*}$. Then the unitary property of $e^{i t \Delta^{2}}$ and Lemma 2.6 imply that

$$
\|u(t)\|_{H^{\beta}} \leq\left\|u_{0}\right\|_{H^{\beta}}+\int_{0}^{t}\|F(u)(s)\|_{H^{\beta}} d s \leq\left\|u_{0}\right\|_{H^{\beta}}+C \int_{0}^{t}\|u(s)\|_{L^{\infty}}^{\nu-1}\|u(s)\|_{H^{\beta}} d s,
$$

for all $0 \leq t<T$. The Gronwall's inequality then yields

$$
\|u(t)\|_{H^{\beta}} \leq\left\|u_{0}\right\|_{H^{\beta}} \exp \left(C \int_{0}^{t}\|u(s)\|_{L^{\infty}}^{\nu-1} d s\right)
$$

for all $0 \leq t<T$. Using (3.2), we see that $\lim \sup \|u(t)\|_{H^{\beta}}<\infty$ as $t \rightarrow T$. This is a contradiction with the blowup alternative in $H^{\beta}$.

\subsection{The case $\gamma>d / 2$}

Let $\beta>\gamma$. If $u_{0} \in H^{\beta}$, then Theorem 1.3 shows that there is a unique maximal solution $u \in C\left([0, T), H^{\beta}\right)$ to $(\mathrm{NL} 4 \mathrm{~S})$. By the uniqueness, we have $T \leq T^{*}$. Suppose $T<T^{*}$. Then

$$
\sup _{0 \leq t \leq T}\|u(t)\|_{H^{\beta}}<\infty
$$

and hence

$$
\sup _{0 \leq t \leq T}\|u(t)\|_{L^{\infty}}<\infty .
$$

This is a contradiction with the fact that limsup $\|u(t)\|_{L^{\infty}}=\infty$ as $t \rightarrow T$. The proof of Theorem 1.6 is now complete.

\section{Ill-posedness}

In this section, we will give the proof of Theorem 1.8 using the technique of [7]. We follow closely the argument of [1]. Let us start with the small dispersion analysis.

\subsection{Small dispersion analysis}

Let us consider for $0<\delta \ll 1$ the following equation

$$
\left\{\begin{aligned}
i \partial_{t} \phi(t, x)+\delta^{4} \Delta^{2} \phi(t, x) & =-\mu|\phi|^{\nu-1} \phi(t, x), \quad(t, x) \in \mathbb{R} \times \mathbb{R}^{d}, \\
\phi(0, x) & =\phi_{0}(x), \quad x \in \mathbb{R}^{d} .
\end{aligned}\right.
$$

Note that (4.1) can be transformed back to (NL4S) by using

$$
u(t, x):=\phi(t, \delta x) .
$$

Lemma 4.1. Let $k>d / 2$ be an integer. If $\nu$ is not an odd integer, then we assume also the additional regularity condition $\nu \geq k+1$. Let $\phi_{0}$ be a Schwartz function. Then there exists $C, c>0$ such that if $0<\delta \leq c$ sufficiently small, then there exists a unique solution $\phi^{(\delta)} \in C\left([-T, T], H^{k}\right)$ of (4.1) with $T=c|\log \delta|^{c}$ satisfying

$$
\left\|\phi^{(\delta)}(t)-\phi^{(0)}(t)\right\|_{H^{k}} \leq C \delta^{3}
$$


for all $|t| \leq c|\log \delta|^{c}$, where

$$
\phi^{(0)}(t, x):=\phi_{0}(x) \exp \left(-i \mu t\left|\phi_{0}(x)\right|^{\nu-1}\right)
$$

is the solution of (4.1) with $\delta=0$.

Proof. We refer to Lemma 2.1 of [7, where the small dispersion analysis is invented to prove the ill-posedness for the nonlinear Schrödinger equation. The proof of Lemma 4.1 is essentially given in Lemma 4.1 of [27], where the author treated the cubic fourth-order Schrödinger equation. The extension to the general power-type nonlinearity here is completely similar. Note that $H^{k}$ with $k>d / 2$ is an algebra.

Remark 4.2. By the same argument as in [7], we can get the following better estimate

$$
\left\|\phi^{(\delta)}(t)-\phi^{(0)}(t)\right\|_{H^{k, k}} \leq C \delta^{3}
$$

for all $|t| \leq c|\log \delta|^{c}$, where $H^{k, k}$ is the weighted Sobolev space

$$
\|\phi\|_{H^{k, k}}:=\sum_{|\alpha|=0}^{k}\left\|\langle x\rangle^{k-|\alpha|} D^{\alpha} \phi\right\|_{L^{2}} .
$$

Now let $\lambda>0$ and set

$$
u^{(\delta, \lambda)}(t, x):=\lambda^{-\frac{4}{\nu-1}} \phi^{(\delta)}\left(\lambda^{-4} t, \lambda^{-1} \delta x\right)
$$

It is easy to see that $u^{(\delta, \lambda)}$ is a solution of (NL4S) with initial data $u^{(\delta, \lambda)}(0)=\lambda^{-\frac{4}{\nu-1}} \phi_{0}\left(\lambda^{-1} \delta x\right)$. We have the following estimate of the initial data $u^{(\delta, \lambda)}(0)$.

Lemma 4.3. Let $\gamma \in \mathbb{R}$ and $0<\lambda \leq \delta \ll 1$. Let $\phi_{0} \in \mathscr{S}$ be such that if $\gamma \leq-d / 2$,

$$
\hat{\phi}_{0}(\xi)=O\left(|\xi|^{\kappa}\right) \text { as } \xi \rightarrow 0
$$

for some $\kappa>-\gamma-d / 2$, where $\hat{\imath}$ is the Fourier transform. Then there exists $C>0$ such that

$$
\left\|u^{(\delta, \lambda)}(0)\right\|_{H^{\gamma}} \leq C \lambda^{\gamma_{c}-\gamma} \delta^{\gamma-d / 2}
$$

The proof of this result follows the same lines as in Section 4 of [7] for the nonlinear Schrödinger equation. We also refer to Lemma 3.3 of [1] for the nonlinear half-wave context.

\subsection{Proof of Theorem 1.8}

We now give the proof of Theorem 1.8. We only consider the case $t \geq 0$, the one for $t<0$ is similar. Let $\epsilon \in(0,1]$ be fixed and set

$$
\lambda^{\gamma_{c}-\gamma} \delta^{\gamma-d / 2}=: \epsilon
$$

equivalently

$$
\lambda=\delta^{\theta}, \text { where } \theta=\frac{d / 2-\gamma}{\gamma_{\mathrm{c}}-\gamma}>1
$$

hence $0<\lambda \leq \delta \ll 1$. We note that we are considering the super-critical range, i.e. $\gamma<\gamma_{\mathrm{c}}$. We will split the proof into several cases. 
The case $0<\gamma<\gamma_{\mathbf{c}}$. We firstly have from Lemma 4.3 and (4.7) that

$$
\left\|u^{(\delta, \lambda)}(0)\right\|_{H^{\gamma}} \leq C \epsilon .
$$

Since the support of $\phi^{(0)}(t, x)$ is independent of $t$, we see that for $t$ large enough, depending on $\gamma$,

$$
\left\|\phi^{(0)}(t)\right\|_{H^{\gamma}} \sim t^{\gamma},
$$

whenever $\gamma \geq 0$ provided either $\nu>1$ is an odd integer or $\gamma \leq \nu-1$ otherwise. Thus for $\delta \ll 1$ and $1 \ll t \leq c|\log \delta|^{c}$, (4.3) implies

$$
\left\|\phi^{(\delta)}(t)\right\|_{H^{\gamma}} \sim t^{\gamma}
$$

Next, using

$$
\left[u^{(\delta, \lambda)}\left(\lambda^{4} t\right)\right]^{\wedge}(\xi)=\lambda^{-\frac{4}{\nu-1}}\left(\lambda \delta^{-1}\right)^{d}\left[\phi^{(\delta)}(t)\right]^{\wedge}\left(\lambda \delta^{-1} \xi\right),
$$

we have

$$
\begin{aligned}
\left\|u^{(\delta, \lambda)}\left(\lambda^{4} t\right)\right\|_{H^{\gamma}}^{2} & =\int\left(1+|\xi|^{2}\right)^{\gamma}\left|\left[u^{(\delta, \lambda)}\left(\lambda^{4} t\right)\right]^{\wedge}(\xi)\right|^{2} d \xi \\
& =\lambda^{-\frac{8}{\nu-1}}\left(\lambda \delta^{-1}\right)^{d} \int\left(1+\left|\lambda^{-1} \delta \xi\right|^{2}\right)^{\gamma}\left|\left[\phi^{(\delta)}(t)\right]^{\wedge}(\xi)\right|^{2} d \xi \\
& \geq \lambda^{-\frac{8}{\nu-1}}\left(\lambda \delta^{-1}\right)^{d-2 \gamma} \int_{|\xi| \geq 1}|\xi|^{2 \gamma}\left|\left[\phi^{(\delta)}(t)\right]^{\wedge}(\xi)\right|^{2} d \xi \\
& \geq \lambda^{-\frac{8}{\nu-1}}\left(\lambda \delta^{-1}\right)^{d-2 \gamma}\left(c\left\|\phi^{(\delta)}(t)\right\|_{H^{\gamma}}^{2}-C\left\|\phi^{(\delta)}(t)\right\|_{L^{2}}^{2}\right) .
\end{aligned}
$$

We also have from (4.8) that $\left\|\phi^{(\delta)}(t)\right\|_{L^{2}} \ll\left\|\phi^{(\delta)}(t)\right\|_{H^{\gamma}}$ for $t \gg 1$. This yields that

$$
\left\|u^{(\delta, \lambda)}\left(\lambda^{4} t\right)\right\|_{H^{\gamma}} \geq c \lambda^{-\frac{4}{\nu-1}}\left(\lambda \delta^{-1}\right)^{d / 2-\gamma}\left\|\phi^{(\delta)}(t)\right\|_{H^{\gamma}} \geq c \epsilon t^{\gamma},
$$

for $1 \ll t \leq c|\log \delta|^{c}$. We now choose $t=c|\log \delta|^{c}$ and pick $\delta>0$ small enough so that

$$
\epsilon t^{\gamma}>\epsilon^{-1}, \quad \lambda^{4} t<\epsilon .
$$

Therefore, for any $\varepsilon>0$, there exists a solution of (NL4S) satisfying

$$
\|u(0)\|_{H^{\gamma}}<\varepsilon, \quad\|u(t)\|_{H^{\gamma}}>\varepsilon^{-1}
$$

for some $t \in(0, \varepsilon)$. Thus for any $t>0$, the solution map $\mathscr{S} \ni u(0) \mapsto u(t)$ for the Cauchy problem (NL4S) fails to be continuous at 0 in the $H^{\gamma}$-topology.

The case $\gamma \leq-d / 2$ and $\gamma<\gamma_{\mathbf{c}}$. Using again Lemma 4.3 and (4.7), we have

$$
\left\|u^{(\delta, \lambda)}(0)\right\|_{H^{\gamma}} \leq C \epsilon
$$

provided $0<\lambda \leq \delta \ll 1$ and $\phi_{0} \in \mathscr{S}$ satisfying

$$
\hat{\phi}_{0}(\xi)=O\left(|\xi|^{\kappa}\right) \text { as } \xi \rightarrow 0,
$$

for some $\kappa>-\gamma-d / 2$. We recall that

$$
\phi^{(0)}(t, x)=\phi_{0}(x) \exp \left(-i \mu t\left|\phi_{0}(x)\right|^{\nu-1}\right) .
$$

It is clear that we can choose $\phi_{0}$ so that

$$
\left|\int \phi^{(0)}(1, x) d x\right| \geq c \text { or }\left|\left[\phi^{(0)}(1)\right]^{\wedge}(0)\right| \geq c,
$$

for some constant $c>0$. Since $\phi^{(0)}(1)$ is rapidly decreasing, the continuity implies that

$$
\left|\left[\phi^{(0)}(1)\right]^{\wedge}(\xi)\right| \geq c,
$$


for $|\xi| \leq c$ with $0<c \ll 1$. Since $H^{k, k}$ controls $L^{1}$ when $k>d / 2$, (4.4) implies

$$
\left|\left[\phi^{(\delta)}(1)\right]^{\wedge}(\xi)-\left[\phi^{(0)}(1)\right]^{\wedge}(\xi)\right| \leq C \delta^{3},
$$

and then

$$
\left|\left[\phi^{(\delta)}(1)\right]^{\wedge}(\xi)\right| \geq c,
$$

for $|\xi| \leq c$ provided $\delta$ is taken small enough. We now have from (4.5) that

$$
u^{(\delta, \lambda)}\left(\lambda^{4}, x\right)=\lambda^{-\frac{4}{\nu-1}} \phi^{(\delta)}\left(1, \lambda^{-1} \delta x\right),
$$

and

$$
\left[u^{(\delta, \lambda)}\left(\lambda^{4}\right)\right]^{\wedge}(\xi)=\lambda^{-\frac{4}{\nu-1}}\left(\lambda \delta^{-1}\right)^{d}\left[\phi^{(\delta)}(1)\right]^{\wedge}\left(\lambda \delta^{-1} \xi\right) .
$$

The estimate (4.9) then yields

$$
\left[u^{(\delta, \lambda)}\left(\lambda^{4}\right)\right]^{\wedge}(\xi) \geq c \lambda^{-\frac{4}{\nu-1}}\left(\lambda \delta^{-1}\right)^{d},
$$

for $|\xi| \leq c \lambda^{-1} \delta$.

In the case $\gamma<-d / 2$, we have from (4.7) that

$$
\left\|u^{(\delta, \lambda)}\left(\lambda^{4}\right)\right\|_{H^{\gamma}} \geq c \lambda^{-\frac{4}{\nu-1}}\left(\lambda \delta^{-1}\right)^{d}=c \epsilon\left(\lambda \delta^{-1}\right)^{\gamma+d / 2} .
$$

Here $0<\lambda \leq \delta \ll 1$, thus $\left(\lambda \delta^{-1}\right)^{\gamma+d / 2} \rightarrow+\infty$. We can choose $\delta$ small enough so that $\lambda \rightarrow 0$ and $\left(\lambda \delta^{-1}\right)^{\gamma+d / 2} \geq \epsilon^{-2}$ or

$$
\left\|u^{(\delta, \lambda)}\left(\lambda^{4}\right)\right\|_{H^{\gamma}} \geq \epsilon^{-1} .
$$

In the case $\gamma=-d / 2$, we have

$$
\begin{aligned}
\left\|u^{(\delta, \lambda)}\left(\lambda^{4}\right)\right\|_{H^{-d / 2}} & \geq c \lambda^{-\frac{4}{\nu-1}}\left(\lambda \delta^{-1}\right)^{d}\left(\int_{|\xi| \leq c \lambda^{-1} \delta}(1+|\xi|)^{-d} d \xi\right)^{1 / 2} \\
& =c \lambda^{-\frac{4}{\nu-1}}\left(\lambda \delta^{-1}\right)^{d}\left(\log \left(c \lambda^{-1} \delta\right)\right)^{1 / 2} \\
& =c \epsilon\left(\log \left(c \lambda^{-1} \delta\right)\right)^{1 / 2}
\end{aligned}
$$

By choosing $\delta$ small enough so that $\lambda \rightarrow 0$ and $\log \left(c \lambda^{-1} \delta\right) \geq \epsilon^{-4}$, we see that

$$
\left\|u^{(\delta, \lambda)}\left(\lambda^{4}\right)\right\|_{H^{-d / 2}} \geq \epsilon^{-1} .
$$

Combining both cases, we see that the solution map fails to be continuous at 0 in $H^{\gamma}$-topology.

The case $\gamma=0<\gamma_{\mathbf{c}}$. Let $a, a^{\prime} \in[1 / 2,2]$. Let $\phi^{(a, \delta)}$ be the solution to (4.1) with initial data

$$
\phi^{(a, \delta)}(0)=a \phi_{0} .
$$

Then, Lemma 4.1 gives

$$
\left\|\phi^{(a, \delta)}(t)-\phi^{(a, 0)}(t)\right\|_{H^{k}} \leq C \delta^{3},
$$

for all $|t| \leq c|\log \delta|^{c}$, where

$$
\phi^{(a, 0)}(t, x)=a \phi_{0}(x) \exp \left(-i \mu a^{\nu-1} t\left|\phi_{0}(x)\right|^{\nu-1}\right)
$$

is the solution of (4.1) with $\delta=0$ and the same initial data as $\phi^{(a, \delta)}$. Note that the constant $C, c$ above can be taken to be independent of $a$ since $a$ belongs to a compact set. We next define

$$
u^{(a, \delta, \lambda)}(t, x):=\lambda^{-\frac{4}{\nu-1}} \phi^{(a, \delta)}\left(\lambda^{-4} t, \lambda^{-1} \delta x\right) .
$$


Thanks to (4.2) and the scaling (1.1), we see that $u^{(a, \delta, \lambda)}$ is also a solution of (NL4S). On the other hand, using (4.11), a direct computation shows that

$$
\left\|\phi^{(a, 0)}(t)-\phi^{\left(a^{\prime}, 0\right)}(t)\right\|_{L^{2}} \geq c>0,
$$

for some time $t$ satisfying $\left|a-a^{\prime}\right|^{-1} \leq t \leq c|\log \delta|^{c}$ provided that $\delta$ is small enough so that $c|\log \delta|^{c} \geq\left|a-a^{\prime}\right|^{-1}$. This estimate and (4.10) yield

$$
\left\|\phi^{(a, \delta)}(t)-\phi^{\left(a^{\prime}, \delta\right)}(t)\right\|_{L^{2}} \geq c,
$$

for all $\left|a-a^{\prime}\right|^{-1} \leq t \leq c|\log \delta|^{c}$. Now, let $\epsilon$ be as in (4.7), i.e.

$$
\lambda^{-\frac{4}{\nu-1}}\left(\lambda \delta^{-1}\right)^{d / 2}=: \epsilon,
$$

or $\lambda=\delta^{\theta}$ with $\theta=\frac{d / 2}{\gamma_{c}}>1$. Moreover, using the fact

$$
\left[u^{(a, \delta, \lambda)}\left(\lambda^{4} t\right)\right]^{\wedge}(\xi)=\lambda^{-\frac{4}{\nu-1}}\left(\lambda \delta^{-1}\right)^{d}\left[\phi^{(a, \delta)}(t)\right]^{\wedge}\left(\lambda \delta^{-1} \xi\right),
$$

we obtain

$$
\left\|u^{(a, \delta, \lambda)}\left(\lambda^{4} t\right)-u^{\left(a^{\prime}, \delta, \lambda\right)}\left(\lambda^{4} t\right)\right\|_{L^{2}}=\lambda^{-\frac{4}{\nu-1}}\left(\lambda \delta^{-1}\right)^{d / 2}\left\|\phi^{(a, \delta)}(t)-\phi^{\left(a^{\prime}, \delta\right)}(t)\right\|_{L^{2}} \geq c \epsilon .
$$

Similarly, using

$$
\left[u^{(a, \delta, \lambda)}(0)\right]^{\wedge}(\xi)=a \lambda^{-\frac{4}{\nu-1}}\left(\lambda \delta^{-1}\right)^{d} \hat{\phi}_{0}\left(\lambda \delta^{-1} \xi\right),
$$

the choice of $\epsilon$ in (4.13) gives

$$
\left\|u^{(a, \delta, \lambda)}(0)\right\|_{L^{2},\left\|u^{\left(a^{\prime}, \delta, \lambda\right)}(0)\right\|_{L^{2}} \leq C \epsilon,}
$$

and

$$
\left\|u^{(a, \delta, \lambda)}(0)-u^{\left(a^{\prime}, \delta, \lambda\right)}(0)\right\|_{L^{2}} \leq C \epsilon\left|a-a^{\prime}\right| .
$$

Since $\left|a-a^{\prime}\right|$ can be arbitrarily small, this shows that for any $0<\epsilon, \sigma<1$ and for any $t>0$, there exist $u_{1}, u_{2}$ solutions of (NL4S) with initial data $u_{1}(0), u_{2}(0) \in \mathscr{S}$ such that

$$
\left\|u_{1}(0)\right\|_{L^{2}},\left\|u_{2}(0)\right\|_{L^{2}} \leq C \epsilon, \quad\left\|u_{1}(0)-u_{2}(0)\right\|_{L^{2}} \leq C \sigma, \quad\left\|u_{1}(t)-u_{2}(t)\right\|_{L^{2}} \geq c \epsilon .
$$

This shows that the solution map fails to be uniformly continuous on $L^{2}$. This completes the proof of Thereom 1.8

\section{Acknowledgments}

The author would like to express his deep gratitude to Prof. Jean-Marc BOUCLET for the kind guidance, encouragement and careful reading of the manuscript.

\section{References}

[1] M. Ben-Artzi, H. Koch, J.C. Saut Disperion estimates for fourth-order Schrödinger equations, C.R.A.S., 330, Série 1, 8792 (2000).

[2] H. Bahouri, J-Y. Chemin, R. Danchin, Fourier analysis and non-linear partial differential equations, A Series of Comprehensive Studies in Mathematis 343, Springer (2011).

[3] J. Bergh, J. Löfstöm, Interpolation spaces, Springer, New York (1976).

[4] T. Cazenave, F. B. Weissler, The Cauchy problem for the critical nonlinear Schrödinger equation in $H^{s}$, Nonlinear Anal. 14, 807-836 (1990). 
[5] T. Cazenave, Semilinear Schrödinger equations, Courant Lecture Notes in Mathematis 10, Courant Institute of Mathematical Sciences, AMS (2003).

[6] Y. Cho, G. Hwang, S. Kwon, S. LeE, Well-posedness and ill-posedness for the cubic fractional Schrödinger equations, Discrete Contin. Dyn. Syst. 35, No. 7, 2863-2880 (2015).

[7] M. Christ, J. Colliander, T. TaO, Ill-posedness for nonlinear Schrödinger and wave equations, https://arxiv.org/abs/math/0311048 (2003).

[8] M. Christ, I. Weinstein, Dispersion of small amplitude solutions of the generalized Korteweg-de Vries equation, J. Funct. Anal. 100, No. 1, 87-109 (1991).

[9] V.D. DinH, Well-posedness of nonlinear fractional Schrödinger and wave equations in Sobolev spaces, https://arxiv.org/abs/1609.06181 (2016).

[10] Strichartz estimates for the fractional Schrödinger and wave equations on compact manifolds without boundary, https://arxiv.org/abs/1609.07305 (2016).

[11] On the Cauchy problem for the nonlinear semi-relativistic equation in Sobolev spaces, https://arxiv.org/abs/1701.00852 (2017).

[12] J. Ginibre, G. Velo, The global Cauchy problem for the nonlinear Klein-Gordon equation, Math. Z. 189, 487-505 (1985).

[13] J. GiniBRE, Introduction aux équations de Schrödinger non linéaires, Cours de DEA 19941995, Paris Onze Edition (1998).

[14] B. Guo, B. WAng, The global Cauchy problem and scattering of solutions for nonlinear Schrödinger equations in $H^{s}$, Differential Integral Equations 15, No. 9, 1073-1083 (2002).

[15] L. Grafakos, S. OH, The Kato-Ponce inequality, Comm. Partial Differential Equations 39, No. 6, 1128-1157 (2014).

[16] C. Hao, L. Hsiao, B. WAng, Well-posedness for the fourth-order Schrödinger equations, J. Math. Anal. Appl. 320, 246265 (2006).

[17] Well-posedness of the Cauchy problem for the fourth-order Schrödinger equations in high dimensions, J. Math. Anal. Appl. 328, 5883 (2007).

[18] Y. Hong, Y. SiRe, On fractional Schrödinger equations in Sobolev spaces, Commun. Pure Appl. Anal. 14, No. 6, 2265-2282 (2015).

[19] Z. HuO, Y. JiA, The Cauchy problem for the fourth-order nonlinear Schrödinger equation related to the vortex filament, J. Differential Equations 214, 135 (2005).

[20] V.I. Karpman, Stabilization of soliton instabilities by higher-order dispersion: Fourth order nonlinear Schrödinger-type equations, Phys. Rev. E 53 (2), 13361339 (1996).

[21] V.I. Karpman, A.G Shagalov, Stability of soliton described by nonlinear Schrödingertype equations with higher-order dispersion, Phys. D 144, 194210 (2000).

[22] T. KATO, On nonlinear Schrödinger equations. II. $H^{\text {s}}$-solutions and unconditional wellposedness, J. Anal. Math. 67, 281-306 (1995).

[23] M. Keel, T. TAO, Endpoint Strichartz estimates, Amer. J. Math. 120, No. 5, 955-980 (1998).

[24] H. Lindblad, C-D. SogGe, On existence and scattering with minimal regularity for semilinear wave equations, J. Funct. Anal. 130, 357-426 (1995).

[25] K. Nakamitsu, T. Ozawa, Nonlinear Schrödinger equation in the Sobolev space of critical order, J. Funct. Anal. 155, 363-380 (1998). 
[26] B. PAusader, Global well-posedness for energy critical fourth-order Schrödinger equations in the radial case, Dynamics of PDE 4, No. 3, 197-225 (2007).

[27] — The cubic fourth-order Schrödinger equation, J. Funct. Anal. 256, 2473-2517 (2009).

[28] J. SEGATA, Well-posedness for the fourth-order nonlinear Schrödinger type equation related to the vortex filament, Differential Integral Equations 16, No. 7, 841864 (2003).

[29] G. Staffilani, The initial value problem for some dispersive differential equations, Dissertation, University of Chicago (1995).

[30] T. TAO, Nonlinear dispersive equations: local and global analysis, CBMS Regional Conference Series in Mathematis 106, AMS (2006).

[31] H. TrieBel, Theory of function spaces, Basel: Birkhäuser (1983).

Institut de Mathématiques de Toulouse, Université Toulouse III Paul Sabatier, 31062 Toulouse Cedex 9, France.

Email: dinhvan.duong@math.univ-toulouse.fr 